\title{
MedienPädagogik
}

www. medienpaed.com

Zeitschrift für

Theorie und Praxis

der Medienbildung

ISSN 1424-3636

\section{Informations- und Kommunikationskompetenz - das «Lesen und Schreiben» der ICT-Kultur}

Nando Stöcklin

\begin{abstract}
Nach der Schrift und dem Buchdruck führen gegenwärtig die elektronischen Medien zu einem dritten grossen Leitmedienwechsel. Ein solcher Leitmedienwechsel führt jeweils zu starken Veränderungen der Kultur. Gemäss Hans Magnus Enzensberger orientiert sich eine neue Kultur zu Beginn immer an der alten, erst später wird sie selbständig und baut einen eigenen Charakter auf. So stammt das Verständnis der Informationskompetenz aus Zeiten, in denen das Buch das Leitmedium war. Massnahmen zur Förderung des kompetenten Umgangs mit Informationen besonders in Bibliotheken sowie Evaluationen der Informationskompetenz von Schülerinnen und Schülern oder von Studierenden basieren auf diesem Verständnis und entsprechenden Modellen. Der aktuelle Leitmedienwechsel von einer vom Buch geprägten Kultur zu einer von Informations- und Kommunikationstechnologien (ICT) - allen voran dem Internet - dominierten Kultur macht ein neues Verständnis der Informationskompetenz notwendig. Im vorliegenden Text werden eine neue Definition, neue Standards und mögliche aktuelle Inhalte zur Förderung von Informationskompetenz systematisch aus kommunikationstheoretischen und soziologischen Betrachtungen des Leitmedienwechsels hergeleitet. Bislang stand bei der Förderung von Informationskompetenz die Rezeption von Texten im Vordergrund. Neu sind dem die Produktion und Übermittlung von TonDokumenten, Videos, Fotos, Grafiken sowie Animationen gleichzustellen. Damit nähert sich der Begriff «Informationskompetenz» stark jenen der Medienkompetenz und der ICT-Kompetenz an.
\end{abstract}

\section{Informationskompetenz in der Buchkultur}

Dem Begriff der Informationskompetenz liegt der englischsprachige Begriff «information literacy» zu Grunde. "Literacy» meint ursprünglich die Lese- und Schreibfertigkeit sowie das Leseverständnis. Seit der Erfindung des modernen Buchdruckes in der Mitte des 15. Jahrhunderts ist Literacy die Voraussetzung, um das Potenzial des Buchdruckes auszuschöpfen und alle Bürger gleichberechtigt an den neuen Errungenschaften wie etwa der späteren neuzeitlichen Demokratie teilhaben zu lassen. 
Mitte der 1970er-Jahre tauchte im englischsprachigen Raum erstmals der Begriff «information literacy» auf und etablierte sich in den folgenden zehn Jahren in der akademischen Fachwelt (Hoffman und Blake 2003; Marcum 2002). 1989 definierte die American Library Association Information Literacy wie folgt:

To be information literate, a person must be able to recognize when information is needed and have the ability to locate, evaluate, and use effectively the needed information.

Im deutschsprachigen Raum haben vor allem die Bibliotheks- und Informationswissenschaften den als «Informationskompetenz» übernommenen Begriff geprägt. Der deutsche Bibliotheksverband e.V. definiert «Informationskompetenz» auf seiner Plattform informationskompetenz. de folgendermassen:

Fähigkeit, die es ermöglicht, bezogen auf ein bestimmtes Problem Informationsbedarf zu erkennen, Informationen zu ermitteln und zu beschaffen sowie Informationen zu bewerten und effektiv zu nutzen.

In der zweiten Hälfte des 20. Jahrhunderts entwickelten andere wissenschaftliche Disziplinen weitere Kompetenz-Begriffe. Die primär aus den Erziehungswissenschaften entstandene Medienpädagogik führte den Begriff «Medienkompetenz» ein. Anfänglich wurde beim Medienkompetenz-Begriff im Vergleich zur Informationskompetenz vor allem audiovisuellen Datentypen Beachtung geschenkt, später den Möglichkeiten des Internets. Ausserdem hat bei der Medienkompetenz die Produktion von eigenen Medienerzeugnissen deutlich mehr Gewicht. Gemäss Dieter Baacke (2007, S. 98 f.) umfasst Medienkompetenz analytische, reflexive und ethische Fähigkeiten zur Medienkritik; informative und instrumentell-qualifikatorische Medienkunde; rezeptiv-anwendende und interaktiv-anbietende Mediennutzung; sowie innovative und kreative Mediengestaltung.

Mit dem Aufkommen des Personal Computers verbreitete sich dann aus dem Umfeld der Informatik der Begriff der ICT-Kompetenz, der stärker die technischen Konzepte von Computern und von dessen Anwendungen betont (Lennon et al. 2003).

In jüngerer Vergangenheit wurden diese Kompetenz-Begriffe vermehrt zusammengefasst, zum Beispiel als «Multimedia-Kompetenz» (Multimedia Literacy) (Institute of Multimedia Literacy 2008) oder als «Meta-Kompetenz» (Metaliteracy) (Mackey und Jacobson 2011). Lippincott (2007) stellt fest, dass Medienkompetenz, Informationskompetenz und ICT-Kompetenz kaum mehr voneinander zu trennen sind. Die Konvergenz der Kompetenzen widerspiegelt die Medienkonvergenz im Internet. 
Informationskompetenz, Medienkompetenz und ICT-Kompetenz lassen sich somit als drei Seiten desselben Würfels sehen:

- Informationskompetenz fokussiert auf die (textlichen) Inhalte

- Medienkompetenz betont die Gefässe

- ICT-Kompetenz blickt vor allem auf die Werkzeuge

In diesem Beitrag soll die Frage beantwortet werden, welche Kompetenzen wir nach dem Leitmedienwechsel von der Buch-zur ICT-Kultur benötigen, um kompetent mit Informationen umgehen zu können. Aufgrund der inhaltlichen Perspektive steht bei der Beantwortung der Frage der Begriff «Informationskompetenz» im Vordergrund. Ein zweiter Grund für die gedankliche Überführung ausgerechnet dieses Begriffs von der Buch- zur ICT-Kultur ist dessen historische Verknüpfung mit dem Medium Buch, die den Leitmedienwechsel zum Internet am deutlichsten von den drei Begriffen sichtbar macht. Sowohl die Medien- als auch die ICT-Kompetenz haben aufgrund ihrer späteren Entstehung implizit den Wechsel teilweise bereits vollzogen, eine explizite Überführung des Begriffs «Informationskompetenz» kann aber nicht nur den Bibliothekswissenschaften, sondern auch der Medienpädagogik und der Informatik Impulse liefern. Und der dritte Grund für die Beschäftigung mit dem Begriff «Informationskompetenz» ist dessen Nähe zum ursprünglichen Begriff «Literacy» als Basis der Schule.

Im Juli 2009 formulierte der deutsche Bibliotheksverband e. V. folgende Standards, die informationskompetente Studierende erfüllen sollten:

1. Die informationskompetenten Studierenden erkennen und formulieren ihren Informationsbedarf und bestimmen Art und Umfang der benötigten Informationen.

2. Die informationskompetenten Studierenden verschaffen sich effizient Zugang zu den benötigten Informationen.

3. Die informationskompetenten Studierenden bewerten die gefundenen Informationen und Quellen und wählen sie für ihren Bedarf aus.

4. Die informationskompetenten Studierenden verarbeiten die gewonnenen Erkenntnisse effektiv und vermitteln sie angepasst an die jeweilige Zielgruppe und mit geeigneten technischen Mitteln.

5. Die informationskompetenten Studierenden sind sich ihrer Verantwortung bei der Informationsnutzung und -weitergabe bewusst.

Definitionen von Informationskompetenz sind nicht explizit auf Print-Informationen eingeschränkt und umfassen etwa auch das Verstehen und Interpretieren von Bildern und Filmen (Bilder und Videos «lesen») und das Erzeugen von Bildern und Videos (Bilder und Videos «schreiben») sowie das Zurechtfinden im Internet. 
Allerdings stehen bei der Förderung von Informationskompetenz in Schulen und Bibliotheken - im Gegensatz zu medienpädagogischen Ansätzen - noch immer Text-Produkte im Vordergrund: "Dem bibliothekarischen Konzept der Informationskompetenz liegt implizit ein Informationsbegriff zugrunde, der sich auf wissenschaftliche, publizierte, gedruckte bzw. elektronische Fachinformation, die systematische Recherche nach sowie die Bewertung, Verwaltung und Nutzung dieser Information reduzieren lässt» (Ingold 2011, S. 57). Schulen konzentrieren sich weiterhin schwergewichtig auf die Förderung des Lesens und Schreibens von sprachlichen Texten; medienpädagogische und informatische Anliegen haben einen schweren Stand. Bibliotheken ihrerseits fokussieren auf die Recherche innerhalb ihrer Institution, indem sie typischerweise Einführungen in die Nutzung von Bibliothekskatalogen und Fachdatenbanken anbieten.

\section{Leitmedienwechsel: Von der Buch- zur ICT-Kultur}

Gegenwärtig befinden wir uns im Übergang von der Buch- zur ICT-Kultur, das heisst, Computer und Internet erweitern die kommunikativen Mittel der vorangegangenen Kultur und lösen das Buch als Leitmedium ab (für vertiefende Informationen siehe Baecker 2007; Giesecke 2002; McLuha, 2001). Ein solcher kultureller Übergang hat starken Einfluss auf unseren Umgang mit Informationen und somit auf die dazu benötigten Kompetenzen.

Der Soziologe Dirk Baecker (2007) untereilt die Menschheitsgeschichte vor der Entwicklung der elektronischen Kommunikationsmittel in drei Epochen:

- Die schriftlose Stammeskultur war von einer oralen Kommunikation geprägt.

- In der Antike kam die Schrift hinzu und übernahm eine zentrale Rolle bei der Informationsweitergabe.

- Die industrialisierte Welt basierte auf dem Buchdruck.

Welche Auswirkungen ein neues Leitmedium auf die verschiedenen Bereiche der Kultur hat, lässt sich am Beispiel des Buchdrucks veranschaulichen. Mit dem Buchdruck waren die Informationen nicht mehr nur auf Klöster beschränkt, sondern konnten sich potenziell an alle Bürger richten. Das Monopol der Kirche auf die Interpretation der Bibel beispielsweise fiel mit Luthers Bibelübersetzung und deren Veröffentlichung. Mit der Aufklärung verbreiteten sich neue Werthaltungen, etwa zu den Rechten der Bürger oder zur Pressefreiheit. Die Revolution des Regierungswesens manifestierte sich beispielsweise in der Unabhängigkeit der USA von Grossbritannien und in der französischen Revolution, die Revolution der Religion in der Reformation und der Religionsfreiheit.

Um das Potenzial des Buchdrucks entfalten zu können, mussten auch die Bürger ausserhalb der Klostermauern des Lesens und Schreibens mächtig und in der Lage sein, das Gelesene auf Basis eines gemeinsamen Wissensstandes zu verstehen. 
Aus diesem Grund entstand ein Bedürfnis nach Schulen für alle. Ausserdem ermöglichte der Buchdruck, dass viele Personen sich gleichzeitig mit denselben Informationen beschäftigten; der bislang individuelle Lernprozess konnte standardisiert werden. Beide Faktoren zusammen führten zur obligatorischen Schule, zur Universität und zur Standardsprache. Bei all diesen Ereignissen war der Buchdruck eine wichtige Voraussetzung.

Ein zentrales Ziel der Schule war bisher, Kindern das Lesen und Schreiben beizubringen, als Voraussetzung, um kompetent mit Informationen umgehen zu können und so als mündige Bürger in unserer Gesellschaft Fuss fassen zu können. Mit den elektronischen Medien und besonders dem Internet haben sich die Voraussetzungen verändert. Lesen und Schreiben ist noch immer von grosser Bedeutung. Allerdings sind Informationen heute nicht mehr hauptsächlich als Print-Produkte erhältlich, sondern vielfach als Videos, Fotos, Grafiken oder Animationen. Zusätzlich haben heute, im Unterschied zu früher, alle Bürger leicht die Möglichkeit, selber Informationen an ein grösseres Publikum zu verbreiten. Der Produktion und Übertragung von Informationen kommt deshalb ein grösserer Stellenwert zu.

\section{Veränderungen beim Umgang mit Informationen}

Gemäss dem Kommunikationstheoretiker Marshall McLuhan (2001) formt sich um eine neue Leittechnologie immer eine neue Kultur. Das Internet als neues Leitmedium dürfte entsprechend einschneidende Veränderungen der Kultur mit sich bringen. Diese Veränderungen lassen sich aus dem veränderten Umgang mit Informationen ableiten:

Beschaffung: Die benötigten Informationen zu beschaffen war in der Buch-Kultur keine triviale Aufgabe. Oft mussten wir Informationen in Form von Büchern, Zeitungen oder Fachzeitschriften kaufen. Deshalb waren Bibliotheken eine wichtige Institution der Buchkultur. In der ICT-Kultur stehen sehr viele Informationen kostenlos und jederzeit zur Verfügung.

Selektion: Bislang haben wenige Personen für viele Menschen Informationen vorselektiert. Buchverlage, Zeitungs-, Radio- und Fernsehredaktionen oder auch Lehrpersonen entschieden, welche Informationen ihr Publikum erreichen sollten. In der ICT-Kultur kommt den persönlichen Netzwerken eine Selektierungsfunktion zu. Im Optimalfall kann der Rezipient von Informationen sowohl in der Buch- als auch in der ICT-Kultur selbst entscheiden, wer für ihn Informationen vorselektieren soll.

Volumen: In der Buchkultur gab es zwar auch ein grosses Informationsangebot, das aber längst nicht so gigantisch war wie jenes der ICT-Kultur. Mit dem riesigen Informationsangebot im Internet finden wir kaum mehr die Zeit, längere Texte oder gar ganze Bücher zu lesen, stattdessen konsumieren wir zunehmend kleinere Informations-Häppchen. 
Nutzungszweck: In der Buchkultur waren vor allem allgemeingültige Informationen von Bedeutung. In der schnelllebigen und immer komplexer werdenden Welt der ICT-Kultur sind besonders Informationen gefragt, die fallbezogen bedeutsam sind. Zeitliche Ausrichtung: Die Berichterstattung in den Massenmedien der Buchkultur erfolgte vergangenheitsbezogen. Im Internet ist eine gegenwartsbezogene Kommunikation beinahe in Echtzeit möglich.

Aktualität: In der ICT-Kultur sind Informationen schnelllebiger und somit rascher veraltet als in der Buchkultur.

Medientyp: In der Buchkultur dominierten sprachliche Text-Informationen. Erst in den letzten Jahrzehnten liessen Fernsehen und Radio auch andere massenmediale Medientypen zu. Mit dem Internet schwindet die Dominanz des Textes immer stärker (Kutteroff und Behrens 2010).

Veröffentlichung: In der Buchkultur konnten zwar theoretisch alle Menschen Informationen an die breite Masse veröffentlichen, de facto musste man aber vielfach entweder in einer entsprechenden Position sein oder entsprechende Kontakte haben. In der ICT-Kultur können alle Internetnutzenden problemlos Informationen veröffentlichen.

Weiternutzung: Gedruckte Informationen konnten in der Buchkultur mit einem gewissen Aufwand abgeschrieben und weiterverbreitet werden. Die digitalen Informationen der ICT-Kultur lassen sich beinahe ohne Aufwand kopieren und weiterverbreiten.

Feedback: Bei der Massenkommunikation der Buchkultur waren Rückmeldungen der Konsumenten nur bedingt möglich und hatten in der Regel keinen Einfluss mehr auf die bereits erfolgte Publikation. Das Internet lässt Rückmeldungen in Echtzeit zu und ist somit ein rückkopplungsstarkes Interaktionssystem (Giesecke 2002, S. 409).

Publikationstypen: Printprodukte beschränken sich auf eine geringe Anzahl Typen, die unterschiedlich beurteilt werden müssen. Das Internet hat bereits eine Vielzahl von Publikationstypen hervorgebracht: Statische Websites, Wikis, Blogs, Microblogs, Foren, Chats, etc. Alle Publikationstypen haben unterschiedliche Eigenschaften.

Management: In der Buchkultur sind viele Informationen schriftlich festgehalten, etwa in Büchern und Zeitungen. Um bei Bedarf auf die Informationen zugreifen zu können, haben wir uns angewöhnt, Informationen, die wir künftig noch benötigen könnten, beiseite zu legen, zum Beispiel im Büchergestell oder in Aktenregistern. Das Internet macht immer mehr Informationen permanent für alle verfügbar und damit örtlich gebundene Informationssammlungen überflüssig. Organisation: Die industrialisierte Welt war hierarchisch organisiert. Vorgesetzte nahmen uns einen wesentlichen Teil der Verantwortung ab und sagten uns, was 
wir wie tun sollten. In der Informationsgesellschaft sind wir zunehmend gefordert, selber Lösungswege zu finden (Giesecke 2005).

Diese veränderten Aspekte des Umgangs mit Informationen machen eine Neudefinition von Informationskompetenz sowie eine neue Gewichtung der Massnahmen zur Förderung von Informationkompetenz notwendig.

\section{Informationskompetenz in der ICT-Kultur}

Jüngere Publikationen zu Informationskompetenz öffnen teilweise den Fokus von statischen Print-Produkten hin zu multimedialen, interaktiven Produkten (Hapke 2007; McNaught 2008; Špiranec und Zorica 2010; Tuominen 2007). Damit nähern sie sich der Medienpädagogik an. Diese neue Sichtweise mündete aber bislang noch nicht in eine systematische Betrachtung der Informationskompetenz unter Berücksichtigung des Leitmedienwechsels und floss noch nicht in Standards zu Informationskompetenz ein und entsprechend auch kaum in die Aktivitäten zur Förderung von Informationskompetenz. In der Folge sollen solche aktualisierten Standards hergeleitet werden.

Aus den neuen Aspekten der Informationsnutzung lassen sich folgende Thesen zu entsprechenden zeitgemässen Kompetenzen ableiten:

Beschaffung: Da in der ICT-Kultur sehr viele Informationen unkompliziert und kostenlos bereitstehen, muss der Beschaffung von Informationen, abgesehen von der Recherche, nur noch eine untergeordnete Beachtung geschenkt werden. So müssen immer seltener z. B. verschiedene Bücher ausfindig gemacht, ausgeliehen und durchstöbert werden auf der Suche nach einer bestimmten Information, stattdessen kann diese im Internet gefunden werden.

Selektierung: Die Vorselektierung von geeigneten Informationen erfolgt in der ICT-Kultur vor allem durch persönliche Netzwerke. Es ist entscheidend, geeignete Netzwerke aufzubauen und sich mit bestehenden Netzwerken zu verbinden.

Volumen: Der Wechsel von umfangreicheren, in sich abgeschlossenen Informationseinheiten zu kleinen Informationshäppchen hat Auswirkungen sowohl auf die Rezeption als auch auf die Publikation von Informationen. Rezipienten wollen sich innert kurzer Zeit durch die Häppchen navigieren und für sie relevante Informationen herausziehen können. Beim Publizieren müssen Informationen kompakt und attraktiv verpackt sein, um in der Fülle von Informationen beachtet zu werden. Wichtig ist auch, ein persönliches Mass zu finden, wie viele Informationen rezipiert und übermittelt werden sollen.

Nutzungszweck: Nebst allgemeingültigen Informationen sind in der ICT-Kultur fallspezifische Informationen von grosser Bedeutung. Fallspezifische Informationen dürften vor allem in entsprechenden Netzwerken bereit stehen und erfordern eine Just-in-Time-Informationsbeschaffung. 
Zeitliche Ausrichtung: Die Kommunikation erfolgt in der ICT-Kultur beinahe in Echtzeit. Konsumenten müssen in der Lage sein, aktuelle Informationen zu finden, in sehr kurzer Zeit zu gewichten und allenfalls in ihren Netzwerken weiterzuleiten. Aktualität: Aufgrund der Schnelllebigkeit von Informationen müssen wir einen grösseren Effort leisten, um unseren Wissensstand ständig aktuell zu halten. Gefragt sind Techniken, Informationen Just in Time zu beschaffen. Bislang stand die Justin-Case-Beschaffung im Vordergrund.

Medientyp: Mit der ICT-Kultur geht die Vormachtstellung von symbolischem Text zu Ende. Wir müssen nicht mehr nur symbolische Text-Dokumente «lesen», «schreiben» und verstehen können, sondern auch ikonische Dokumente, also Bilder, interaktive Grafiken, Videos, Ton-Dokumente und Animationen sowie vielleicht gar symbolischen Code wie HTML oder CSS.

Veröffentlichung: Neu können alle Internetnutzer problemlos Informationen publizieren. Informationen publizieren und in der Öffentlichkeit kommunizieren zu lernen ist deshalb ebenso wichtig wie Informationen rezipieren zu lernen.

Weiternutzung: Da sich Informationen heute problemlos durch Copy/Paste vervielfältigen lassen, müssen wir Bescheid wissen über rechtliche und akademische Vorgaben wie Urheberrechte und korrektes Zitieren.

Feedback: Viele Informationen im Internet können wir in der ICT-Kultur kommentieren, umgekehrt können von uns publizierte Mitteilungen kommentiert werden. Es ist von grosser Wichtigkeit, den sinnvollen Umgang mit Rückmeldungen zu erlernen.

Publikationstypen: Publikationstypen wie Blogbeiträge, Twittermeldungen oder Wikiseiten haben unterschiedliche Eigenschaften. Beispielsweise zur Wahl des Publikationstyps zur Kommunikation oder bei der Einschätzung der Qualität ist es unerlässlich, die wesentlichen Eigenschaften zu kennen.

Management: Unsere Angewohnheit, Informationen beiseite zu legen, weil wir sie evtl. einmal benötigen könnten, hilft uns in der schnelllebigen ICT-Kultur wenig. Entsprechend dürfte das Management von Informationen an Bedeutung verlieren und die Informationsrecherche noch weiter an Bedeutung gewinnen. Organisation: In der ICT-Kultur ist Selbstverantwortung eine zentrale Eigenschaft. Die hierarchische Arbeit weicht der Arbeit in Gruppen und Netzwerken. Wir müssen uns selbst stets auf dem Laufenden halten, müssen in der Lage sein, uns mit geeigneten Personen zu vernetzen und müssen kompetent mit Informationen umgehen können. Die Förderung der Informationskompetenz wird deshalb umso wichtiger.

Einzelne neue Aspekte wie die Schnelllebigkeit sowie das veränderte Management von Informationen lassen sich gut in der bisherigen Definition von Informationskompetenz unterbringen und erfordern bloss neue Inhalte bei der Förderung. 
Andere Eigenschaften wie die explizite Berücksichtigung unterschiedlicher Datentypen und die Produktion und Übermittlung von Informationen machen eine neue Gewichtung notwendig. Die Produktion und Übermittlung sind in der ICT-Kultur gleichwertig zu behandeln wie die Rezeption, und Bilder, Videos und Ton-Dokumente gleichwertig wie sprachlicher Text.

War die Förderung der Informationskompetenz bislang vor allem auf den rezeptiven Erschliessungsprozess von printbasierten Informationen ausgelegt, kommen jetzt zwei neue Dimensionen hinzu (s. Abb. 1): Neben der Rezeption werden die übrigen Elemente der Kommunikation, also die Produktion und Übertragung von Informationen immer wichtiger und Print-Produkte sind in unserem multimedialen Umfeld nur noch ein Datentyp von mehreren. Damit nähert sich die Förderung der Informationskompetenz sowohl der Medienpädagogik als auch der Informatik an. Die Medienpädagogik kann etwa die differenzierende Analyse der unterschiedlichen Datentypen oder Wissen zur Produktion von Ton-Dokumenten oder Videos einbringen, die Informatik die technischen Konzepte der verschiedenen Dateitypen sowie Wissen zur Funktionsweise des Internets und dessen Anwendungsdiensten.

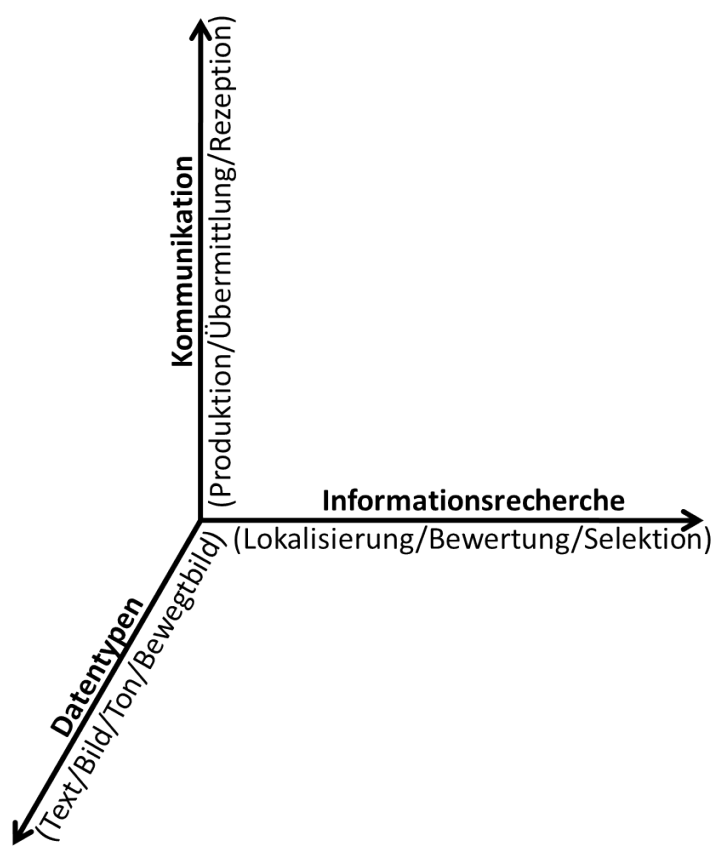

Abbildung 1: Die drei Dimensionen der Informationskompetenz in der ICT-Kultur. 
Um den veränderten Bedingungen gerecht zu werden, bietet sich folgende neue Definition von Informationskompetenz an:

Fähigkeit, die es ermöglicht, Informationen effizient und in geeigneten Medientypen zu ermitteln, selektieren und beschaffen; zu verarbeiten, umzuwandeln und zu erzeugen; sowie über geeignete Kanäle zu kommunizieren.

Aus der vorgeschlagenen Definition lassen sich folgende Standards für Studierende sowie Schülerinnen und Schüler ableiten. Fragen zur Entwicklung von Standards beispielsweise aus der Medienpädagogik (s. etwa Herzig und Grafe 2010) werden bewusst ausgeblendet; stattdessen orientiert sich der Vorschlag der besseren inhaltlichen Vergleichbarkeit wegen am Kompetenzbegriff der bisherigen Informationskompetenz-Standards. Es wird auch keine Aufteilung in Mindest-, Regel- und Höchststandards vorgenommen.

1. Die informationskompetenten Studierenden erkennen ihren stetigen, wie auch situativen Informationsbedarf und bestimmen Art und Umfang der benötigten Informationen.

2. Die informationskompetenten Studierenden verschaffen sich effizient Zugang zu Informationen, beurteilen diese und wählen geeignete Informationen für ihren Bedarf aus.

3. Die informationskompetenten Studierenden erkennen ihren stetigen und situativen Übermittlungsbedarf sowie geeignete Kanäle und bestimmen Art und Umfang der zu übermittelnden Informationen.

4. Die informationskompetenten Studierenden verwenden die Informationen praktisch oder bereiten sie effizient und effektiv in geeigneter Art und geeignetem Umfang auf und übermitteln sie.

5. Die informationskompetenten Studierenden sind sich bei der Informationsnutzung und -weitergabe der Verantwortung gegenüber anderen und sich selbst bewusst.

Die Förderung der Informationskompetenz kann nebst Lesen, Schreiben und Rechnen beispielsweise folgende Themen umfassen: 


\begin{tabular}{|l|l|}
\hline $\begin{array}{l}\text { Informationen } \\
\text { beschaffen }\end{array}$ & $\begin{array}{l}\text { Anwenderwissen: horizontale vs. vertikale Suchmaschinen nutzen; RSS-Feeds und } \\
\text { Mailinglisten finden und abonnieren; Microblogging-Dienste nutzen; persönliche Netz- } \\
\text { werke aufbauen und gezielt nutzen, Verlässlichkeit von Informationen abschätzen z. B. } \\
\text { anhand der URL }\end{array}$ \\
& $\begin{array}{l}\text { Orientierungswissen: Begrifflichkeiten wie Ausbeute und Präzision, Katalog vs. Index } \\
\text { vs. Tags kennen; stetige vs. problembasierte Informierung, Just in Case vs. Just in Time } \\
\text { unterscheiden; Alterung von Informationen berücksichtigen; Umgang mit Informations- } \\
\text { fülle; Open-Access-Prinzip kennen }\end{array}$ \\
\hline $\begin{array}{l}\text { Informationen } \\
\text { verändern und } \\
\text { produzieren }\end{array}$ & $\begin{array}{l}\text { Anwenderwissen: Videos, Ton-Dokumente, Vektor- und Rastergrafiken, Animationen } \\
\text { sowie interaktive Infografiken erstellen und bearbeiten; Informationen zu konzisen } \\
\text { Häppchen verarbeiten; Zitationsregeln anwenden; Werkzeuge zur Kollaboration nutzen }\end{array}$ \\
& $\begin{array}{l}\text { Orientierungswissen: Urheber- und Nutzungsrecht verstehen; Plagiate vermeiden; Wir- } \\
\text { kung von Bildern und Schreibstilen kennen; Eigenschaften von Medientypen kennen; } \\
\text { Interaktivitätstaxonomie kennen }\end{array}$ \\
\hline $\begin{array}{l}\text { Informationen } \\
\text { übermitteln }\end{array}$ & $\begin{array}{l}\text { Anwenderwissen: Grundlagen des Webpublishing (HTML, CSS, Navigationsstruktur) } \\
\text { beherrschen; verschiedene Netzwerke aufbauen und betreuen (Soziale Netzwerke, } \\
\text { Blogs, Microblogs, Mailinglisten, ...); Datensicherheit (Backup, https) umsetzen }\end{array}$ \\
\hline $\begin{array}{l}\text { Orientierungswissen: Eigenschaften verschiedener Netzwerke kennen; Bild-Auflösung } \\
\text { verstehen; Design-Prinzipien kennen; Persönlichkeitsrechte und freie Lizenzen verste- } \\
\text { hen; Eigenarten von verschiedenen Kanälen (E-Mail, öffentliches Web, analog/digital) } \\
\text { kennen; mit Konflikten in der Öffentlichkeit umgehen können; Gefahren durch Cyber- } \\
\text { mobbing verstehen }\end{array}$ \\
\hline
\end{tabular}

\section{Ausblick}

Heute werden in der Schule mit Lesen, Schreiben und Rechnen einige Grundlagen gefördert, um mit Informationen kompetent umgehen zu können. Darüber hinaus werden Aspekte der Kommunikation gefördert und teilweise hielten Massnahmen zur Medienbildung oder zur informatischen Bildung Einzug in die Schulen. Bibliotheken bieten zusätzliche Weiterbildungen vor allem zu einem Teilgebiet des Information Retrievals an. Die überwiegende Mehrheit der Informationskompetenz-Standards der ICT-Kultur wird in der formellen Bildung aber noch nicht gezielt angegangen.

Die zentrale Herausforderung der Buchkultur war es, möglichst allen Menschen das Lesen und Schreiben beizubringen sowie das Leseverständnis zu fördern, damit sie die Vorzüge der Bücher und anderer Print-Produkte nutzen konnten. Kurz: Die Bürger mussten kompetent mit Informationen umgehen können, also informationskompetent sein. 
Auch in der ICT-Kultur lautet die Herausforderung, möglichst alle Menschen informationskompetent zu machen, damit sie die Vorzüge der Informations- und Kommunikationstechnologie nutzen können. Informationskompetenz in der ICT-Kultur ist aber ungleich komplexer und umfassender als Informationskompetenz in der Buch-Kultur. Um dabei der Bedeutung der Kommunikation angemessen Gewicht zu verleihen, bietet sich heute der Begriff «Informations- und Kommunikationskompetenz» an.

Es gilt nun, die erforderlichen Kompetenzen gezielt zu fördern. Hierzu können wir auf fundierte Vorarbeiten der Medienpädagogik und der Informatik zurückgreifen. Doch selbst dann ist die Herausforderung nicht zu unterschätzen. Beispielsweise die konsequente Umstellung vom Just-in-Case-Bildungssystem zum Just-in-TimeSystem hat einschneidende Veränderungen zur Folge.

Die obligatorische Schule war die Antwort auf die neuen Anforderungen der Buchkultur an die Bildung. Mit den veränderten Anforderungen aufgrund des Leitmedienwechsels vom Buch zum Internet wurde das Fundament des Bildungssystems, die notwendige Informationskompetenz, stark verändert. Es muss ein neues Bildungsverständnis entwickelt werden, das auf das neue Fundament passt, ein Bildungssystem, in dem die allermeisten Menschen - analog zur Alphabetisierung der Buchkultur - zu einer kompetenten Nutzung der Informations- und Kommunikationstechnologie befähigt werden können.

\section{Literatur}

Baacke, Dieter. 2007 (1. Aufl. 1997). Medienpädagogik. Tübingen: Max Niemeyer. Baecker, Dirk. 2007. Studien zur nächsten Gesellschaft. Frankfurt a. M.: Suhrkamp. Enzensberger, Hans Magnus. 1988. Mittelmass und Wahn. Frankfurt a. M.: Suhrkamp.

Giesecke, Michael. 2002. Von den Mythen der Buchkultur zu den Visionen der Informationsgesellschaft. Frankfurt a. M.: Suhrkamp.

Giesecke, Michael. 2005. "Auf der Suche nach posttypographischen Bildungsidealen.» Zeitschrift für Pädagogik 1: 14-29.

Hapke, Thomas. 2007: «Informationskompetenz 2.0 und das Verschwinden des Nutzers.» Bibliothek: Forschung und Praxis 31 (2): 137-149.

Herzig, Bardo und Silke Grafe. 2010. «Entwicklung von Bildungsstandards für die Medienbildung - Grundlagen und Beispiele.» In Jahrbuch Medienpädagogik 8 , hrsg. v. Bardo Herzig, Dorothee M. Meister, Heinz Moser und Horst Niesyto, 103-120. Wiesbaden: VS Verlag für Sozialwissenschaften.

Hoffman, Mark und Jonathan Blake. 2003. "Computer literacy: today and tomorrow.» Journal of Computing Sciences in Colleges 18(5): 233. Consortium for Computing Sciences in Colleges. 
Ingold, Marianne. 2011. «Information als Gegenstand von Informationskompetenz. Eine Begriffsanalyse.» Berliner Handreichungen zur Bibliotheks- und Informationswissenschaft Heft 294: http://edoc.hu-berlin.de/series/berliner-handreichungen/2011-294/PDF/294.pdf (22.6.2012).

Institute of Multimedia Literacy. 2008. Introduction to Multimedia Scholarship Companion Handbook for MDA 140 offered in conjunction with Multimedia in the Core Multimedia Across the College Programs. Los Angeles.

Kutteroff, Albrecht und Peter Behrens. 2010. JIM 2010: Jugend, Information, (Multi-)Media. Basisstudie zum Medienumgang 12- bis 19-Jähriger in Deutschland. Stuttgart: Medienpädagogischer Forschungsverbund Südwest.

Lennon, Marylou, Irwin Kirsch, Matthias Von Davier, Michael Wagner und Kentaro Yamamoto. 2003. Feasibility Study for the PISA ICT Literacy Assessment. ACER.

Lippincott, Joan K. 2007. Student Content Creators: Convergence of Literacies. Educause Review 42 (6): 16-17.

Mackey, Thomas P. und Trudi E. Jacobson. 2011. «Reframing Information Literacy as a Metaliteracy.» College \& Research Libraries 72 (1): 62-78.

Marcum, James W. 2002. "Rethinking Information Literacy.» The Library Quarterly $72(1): 1$.

McLuhan, Marshall. 2001. Das Medium ist die Botschaft - The Medium is the Message. Hrsg. u. übers. v. Martin Baltes, Fritz Boehler, Rainer Höltschl u. a. Dresden: Verlag der Kunst.

McNaught, Carmel. 2008. "Information Literacy in the 21 ${ }^{\text {st }}$ Century.» In Encyclopedia of Information Technology Curriculum Integration, hrsg. v. Lawrence A. Tomei, 406-412. doi:10.4018/978-1-59904-881-9 (22.6.2012).

Špiranec, Sonja und Mihaela B. Zorica. 2010. «Information literacy 2.0: Hype or discourse refinement?» Journal of Documentation 66 (1): 140-153.

Tuominen, Kimmo. 2007. «Information Literacy 2.0.» Signum 40 (5), 6-12. 